spond to specific questions and to attempt to demystify the process generally. The interests of FDA, consumers, and industry are all best served by regulation that is transparent and well understood.

Henry I. Miller Director

Office of Biotechnology

Food and Drug

Administration

Bethesda, MD 20785

We thank Drs. Krawiec and Miller for their clarifications-the need for which only underscores the problems vaccine development present. While we thank Miller for his charity-the misconstruction of IND and PLA requirements is rather more than a nuance-we must sympathize with neophytes seeking FDA guidance for the first time: $a$ dozen telephone calls logged to FDA's individual centers and the Office of Biotechnology failed to elicit any substantive help on vaccine regulation-certainly nothing as concise as the one Miller offers here.-The Editors

\section{INFORMATION, PLEASE}

\section{To the editor:}

In his recent report (Bio/Technology 10:752, "What form for Eurobioinformatics?") John Hodgson offers his view of the politics associated with the efforts to design and implement the European Bioinformatics Institute (EBI). While political news may be fun to read, whether it's accurate or not, Hodgson misses the essential point of why the EBI must exist and very soon. That is to ensure European access to, influence on, and expertise for making the best use of the biological information critical to both basic research efforts and the biotechnology industry.

The EMBL Data Library has for over a decade creditably collected and delivered a variety of sequence and related biological information, with much of its effort focused on scientific annotation and data processing and distribution. Continuing this traditional role will certainly require increased funding, but this alone will not ensure that biologists have access to the new forms of information they will need to solve tomorrow's research and biotechnology problems. This will only be possible if the EBI and its collaborators invest in applying the latest computer and informatics technology to managing biological information and in keeping pace with biological research advances. The technology and the science are changing too rapidly to imagine that yesterday's solutions will be viable for long. The information of the near future will be remarkably rich and diverse (not simply text, not simply sequence or map location, but integrated images, structures and function), geographically distributed (available over the international networks) and maintained by numerous independent researchers. There is no one solution for making this information maximally useful except the continuing process of innovation in support of service. Such service and innovation require stable long-term support.

The EMBL Data Library's current funding and facilities do not permit this investment to any significant degree. This is also true for many other bioinformatics resources around the world, and has left some of these moldering away in dusty corners with antiquated solutions to irrelevant problems. Our proposed EBI will continue with the current tasks of the Data Library including its international collaboration (not competition) with the NCBI and others, especially scientists in European academe and industry, but it would be irresponsible of us not to plan and invest for the future. We believe that the EMBL Council and the EC recognise the importance of ensuring the future of the Data Library and will find suitable funding despite economic constraints. However, we have never suggested that the EBI will cost an order of magnitude more than the current Data Library, and we have always planned to seek support from a variety of sources including the EC, EMBL and industry. This will also ensure that all constituencies of the EBI, including users of its services, have a voice in its governance.

Howard Bilofsky

Executive Planning Officer

European Bioinformatics Institute EMBL Heidelberg, Germany

A European Bioinformatics Institute remains one-but at this stage only one of several-options for European bioinformatics. Ultimately, the community at large must weigh the scientific arguments for and against all of these options though open discussion in public foraincluding Bio/Technology.-The Editors

\section{DNA Flngerprinting: MAAPing out a RAPD Redefinition?}

\section{To the editor:}

The advent of a novel strategy for DNA fingerprinting that uses a single oligonucleotide to prime arbitrary segments of a DNA template to produce a characteristic set of amplified fragments promises to be of immense value in the analysis of genetic relationships. ${ }^{1}$ The idea was conceived and developed by several laboratories, ${ }^{24}$ each using markedly differentamplification and DNA separation procedures, as well as primers of different length. Fingerprint complexity varies from very simple, and thus ideal for genomic mapping, ${ }^{2}$ to highly complex and more suitable for fingerprinting. ${ }^{4}$ However, with this novel strategy came different and sometimes incorrect terminologies. The terms Random Amplified Polymorphic DNA (RAPD) ${ }^{2}$ and Amplification Fragment Length Polymorphism (AFLP) ${ }^{4}$ are used to describe polymorphisms, while Arbitrarily Primed Polymerase Chain Reaction (APPCR $)^{3}$ and DNA amplification fingerprinting (DAF) ${ }^{4}$ describe the actual strategy. AP-PCR conveys a closer description of the amplification strategy, however the use of a single primer to target both DNA strands differs notably from the PCR, in which two primers independently target each DNA strand in a reaction that strives for specificity. What then is the correct term to portray this mapping-fingerprinting procedure? We are all tired of remembering the myriad acronyms that describe the many emerging approaches in molecular biology. If we are to tolerate one more, let us find a suitably correct term, one that encompasses each variation of the overall strategy. We suggest the term Multiple Arbitrary Amplicon Profiling (MAAP) to describe its underlying characteristics: the multiple, arbitrary nature of targeted sites and the amplification of a range of characteristic DNA products. Since MAAP can be used to place markers in a genetic map the acronym may prove appropriate. While proponents of each terminology may resist a new acronym, it is nevertheless important to reach consensus. Because of the RAPD expansion of this new and exciting field we should not delay a decision. Tous pour un, un pour tous!

Gustavo Caetano-Anollés Brant J. Bassam

Peter M.Gresshoff Plant Molecular GeneticsInstitute for Agriculture \& Center for Legume Research

University of Tennessee

Knoxville, TN 27901-1071

1. P. Hendrick, Nature 355:679-680 (1992).

2. J.G.K. Williams, A.R. Kubelik, K.J. Livak, J.A. Rafalski, S.F. Tingey, Nucl. Acids Res. 18:6531-6535 (1990).

3. J. Welsh, M. McClelland, Nucl. Acids Res. 18:7213-7218 (1990).

4. G. Caetano-Anollés, B.J. Bassam, P.M. Gresshoff, Bio/Technology 9:553-557 (1991). 